ORIGINAL ARTICLE

\title{
Vulvovaginal candida in a young sexually active population: prevalence and association with oro-genital sex and frequent pain at intercourse
}

\author{
E Rylander, A-L Berglund, C Krassny, B Petrini
}

Sex Transm Infect 2004;80:54-57. doi: 10.1136/sti.2003.004192

See end of article for authors' affiliations

Correspondence to:

Eva Rylander, Division of Obstetrics and

Gynaecology, Karolinska

Institutet Danderyd

Hospital, SE-182 88

Stockholm, Sweden;

eva.rylander@kids.ki.se

Accepted for publication 6 August 2003
Objective: To study the prevalence of vulvovaginal candida among sexually active adolescents. To determine past and present symptoms, including pain at intercourse and potential behavioural risk factors associated with vulvovaginal candidiasis.

Methods: At an adolescent centre, 219 sexually active women who underwent genital examination, also completed a questionnaire on a history of genital symptoms and infections, sexual and hygiene habits, and the use of contraceptives. Symptoms and clinical signs were registered. Vaginal samples were analysed for candida species and urine for Chlamydia trachomatis.

Results: Candida culture was positive in $42 \%$ of the women and only $15 \%$ were asymptomatic. A history of recurrent candidiasis was given by $22 \%$. Frequent pain at intercourse was reported by $24 \%$ and frequent oro-genital sex by $42 \%$ of the women. Frequent pain at intercourse was significantly associated with both the growth of candida and a history of recurrent candidiasis. Oro-genital sex was an independent risk factor for the growth of candida.

Conclusion: In sexually active adolescents, who underwent genital examination, candida cultures were positive in $42 \%$. The habit of frequent oro-genital sex was associated with the growth of candida. Pain at intercourse was associated with the growth of candida and recurrent candidiasis. l: $\mathrm{n}$ recent years, it has become more common for young women to consult adolescent health centres or STD clinics because of vulvar discomfort and burning pain during and after intercourse. ${ }^{12}$ In the future, some of these women will probably develop a chronic pain syndrome, vulvar vestibulitis (VVS), which is a growing problem. ${ }^{3}$ An association between VVS and earlier recurrent vulvovaginal candidiasis has been observed. ${ }^{45}$

During their lifetime, $75 \%$ of the female population will have at least one episode of candida vulvovaginitis and 40$50 \%$ will have recurrent episodes. ${ }^{67}$ An increase in the incidence of vaginal candidiasis has been reported $^{7}$ but hitherto there has not been any study dealing with the prevalence among sexually active adolescents.

The aim of this study was to assess the prevalence of vulvovaginal growth of candida in sexually active young women and to determine past and current symptoms, including pain at intercourse, and some potential risk factors associated with a candida infection.

\section{METHODS}

The study was carried out in the year 2000 at a single adolescent health centre in Stockholm, Sweden. The purpose of adolescent health centres, which are run by midwives, is primarily to provide counselling on sexual matters and to prescribe contraceptives. Sexually active women for whom a genital examination was indicated, were requested to participate. The reasons for genital examination were (1) the prescribing of oral contraceptives and screening for STD $(53 \%)$, and (2) the presence of any genital symptoms (47\%). The indications for genital examination during the study period did not differ from those of other periods at the health centre.

The purpose of the visit and any genital symptoms were registered. The women were asked to complete a short questionnaire on the use of oral contraceptives (OC) and condoms, age at first intercourse, duration of regular sex life, frequency of intercourse, number of partners, use of antibiotics, consumption of alcohol and sweets, use of tobacco, personal hygiene, and a history of genital infections. Past episodes of candidiasis were based on a history of vulvar pruritus and a curdy, whitish discharge that disappeared after local or systemic antifungal medication. In this study, three episodes or more are referred to as recurrent candidiasis. Because of the young age of the participants, we did not use the criteria for chronic candidiasis (four episodes or more per year). A history of vulvar itching, fissures, abnormal discharge and frequent or regular pain at intercourse, was also registered. Discoloured, foul smelling, whitish, thick and voluminous discharge was defined as abnormal. The use of sanitary pads between periods, thongs, and shaving of pubic hair was registered as never, sometimes, or frequent.

At the genital examination, performed by the midwife, the presence of abnormal discharge, erythematous mucosa, and fissures was recorded. Wet mounts were not examined since Swedish midwives are generally not trained to perform light microscopy. Vaginal samples were collected by cotton swabs for fungal culture. The fungal cultures were plated on CHROM-agar and glucose blood agar directly from the vaginal swabs, usually on the same day. Yeasts were identified by standard methods. " Chlamydia trachomatis was analysed when indicated or if requested by the patient. Thus, urine specimens from 155 women were analysed for chlamydia by polymerase chain reaction (PCR). Cell samples from the cervix and vagina were collected for analysis of the human papillomavirus (HPV) and other organisms of the vaginal microflora, the results of which will be presented elsewhere. Since there is a very low prevalence of gonorrhoea in Sweden, only a few samples were tested.

\section{Statistical analysis}

The $\chi^{2}$ test was used to assess differences between candida positive and negative subjects regarding vulvovaginal 
symptoms and signs, as well as sexual and hygiene habits. A logistic regression model (backwards stepwise) was used to evaluate the association between the growth of candida and behavioural factors mentioned. The same method was employed to evaluate the independent effects of these variables on pain at intercourse.

The study was approved by the local ethics committee of the Karolinska Hospital. Participation was voluntary and anonymous.

\section{RESULTS}

In total, 220 sexually active women were examined and completed the questionnaire. All of the women who were requested to participate in the study consented but one had to be excluded since her sample for fungal culture was lost. The mean age of the women was 19 (range 12-22) years. The mean age at the debut of sexual intercourse was 16 years (range 12-20).

$C$ albicans species were isolated in 90 out of 219 samples and $C$ glabrata in three.

Of 155 urinary samples, only four $(2.6 \%)$ were positive for $C$ trachomatis by PCR. All samples for $N$ gonorrhoeae tested negative. History of previous genital infections is shown in table 1.

Of 93 women with a positive candida culture, 79 (85\%) complained of current itching, discharge, fissures, or vulvar pain. Thus, only $14(15 \%)$ had an asymptomatic candida infection. Altogether, $6 \%$ of the participating women were asymptomatic candida carriers.

Past and present complaints of vulvar pruritus and discharge as well as clinical signs of vulvar erythema, fissures, and abnormal discharge were significantly more common in women with a positive candida culture than in those with a negative culture $(\mathrm{p}<0,001)$ (table 2$)$. A history of pain at intercourse was given by 52 (24\%) of 217 women. Pain at intercourse was associated with a positive candida culture $(\mathrm{p}<0.004)$ (table 2$)$. A history of recurrent candidiasis was also associated with pain at intercourse $(p<0.01)$.

In a multivariate logistic regression model, with pain at intercourse as dependent variable, and positive candida culture, current OC use, OC for 3 years or longer, regular intercourse more than 4 years, intercourse more than twice a week, and frequent oro-genital sex as independent variables, only positive candida culture was significantly associated with pain at intercourse, $\mathrm{OR}=2.49$ (CI 1.26 to 4.94).

Oro-genital sex was practised occasionally by 111 of the women and frequently by 88 . Only 15 out of 214 women reported never having had oral sex. There was a significant association between growth of candida and frequent practice of oro-genital sex $(\mathrm{p}=0.02)$ (table 3$)$.

A history of recurrent candidiasis was twice as common among women who had used OC for at least 3 years $(31 \%)$ compared to women who had used OC for a shorter period or not at all $(14 \%)(\mathrm{p}=0.004)$.

There was a trend towards a relation $(p=0.06)$ between positive candida culture and frequent sweet consumption, but no association with the other behavioural factors (table 3).

Among a set of factors including frequent sexual intercourse, sexual intercourse for 4 years, current use of OC, frequent use of condoms, use of antibiotics more than three times during the past 2 years, and frequent oro-genital sex, only frequent oro-genital sex remained significantly associated with positive candida culture, $\mathrm{OR}=1.97(\mathrm{CI}=1.03$ to 3.78).

\section{DISCUSSION}

It is reasonable to believe that young women with genital symptoms consult adolescent health centres more often than women without such symptoms. Thus, the present study is not considered to be population based. The prevalence of isolated candida species on culture in this young sexually active cohort was higher $(42 \%)$ than any previously reported figure. Candida was diagnosed in 30\% of women 15-19 years of age at an STD clinic in Seattle and in $23-31 \%$ of women at genitourinary clinics. ${ }^{10}{ }^{11}$ In a retrospective microscopy study of Papanicolaou smears, it was observed that candida is most common in young women (20 years or less). ${ }^{8}$

In order to determine the prevalence of subjects with asymptomatic candida, it is necessary to register current symptoms and signs in combination with culture or wet mount analysis of yeast. Only few such studies have been published $^{10}{ }_{12}$ except for the present one, which shows only $6 \%$ asymptomatic growth of candida.

According to reported history, candidiasis was the most common genital infection in our study cohort (60\%). Episodes of foul smelling discharge, probably reflecting bacterial vaginosis, were reported by $32 \%$ of the women. The prevalence of chlamydia and HPV according to the history, is in accordance with earlier similar studies of Swedish women. ${ }^{12}$ Chlamydia culture was positive in $2.6 \%$ of our study cohort, a figure much lower than earlier observed at the adolescent centre. Interestingly enough, in a study by Eckerts et al, positive candida culture associated negatively with chlamydia infection. ${ }^{10}$ Moreover, in a survey on female sex workers, chlamydia was observed in $4,5 \%$ of women with candida and in $20 \%$ in women with trichomonas. ${ }^{13}$ Hypothetically, after antibiotic treatment for any reason, women might have been cured of chlamydia but acquired candida instead.

Our result, showing a significant association between orogenital sex and growth of candida infection, is supported by previous studies. ${ }^{14-17}$ One reason for such a correlation might be contact transmission..$^{18}$ One third of the adult population is said to harbour oral Candida albicans. ${ }^{17}{ }^{18}$ It has also been proposed that saliva may promote growth of candida through moistening and irritation of the vulvar mucosa ${ }^{14}$ or by changing the local immunological state. ${ }^{17}$

Spinillo et al observed that women on OC had a tendency to have recurrent candidiasis. ${ }^{19}$ Our result, showing that recurrent candida was more common among long time OC users, supports their observation.

Table 1 History of genital infections in sexually active adolescents

\begin{tabular}{lll}
\hline & Prevalence of previous infection & Number of answers \\
\hline Vulvovaginal candida at least once & $128(60 \%)$ & 214 \\
Recurrent candidiasis* & $45(22 \%)$ & 207 \\
Chlamydia trachomatis & $19(9 \%)$ & 202 \\
HPV & $23(11 \%)$ & 205 \\
HSV & $4(2 \%)$ & 199 \\
Gonorrhoea & 0 & 199 \\
Foul smelling discharge & $65(32 \%)$ & 203 \\
\hline
\end{tabular}

*A history of vulvovaginal candidiasis three times or more. 
Table 2 Vulvovaginal symptoms and signs in sexually active adolescents

\begin{tabular}{|c|c|c|c|c|c|}
\hline & \multicolumn{2}{|l|}{ Candida } & \multirow[b]{2}{*}{ Number of answers } & \multirow[b]{2}{*}{$\chi^{2}$} & \multirow[b]{2}{*}{ p Value } \\
\hline & Positive & Negative & & & \\
\hline \multicolumn{6}{|c|}{ History of recurrent symptoms } \\
\hline Itching & $52(60 \%)$ & $44(36 \%)$ & 207 & 11.74 & $<0.001$ \\
\hline Fissures & $38(45 \%)$ & $27(23 \%)$ & 203 & 11.50 & $<0.001$ \\
\hline Pain at intercourse & $31(34 \%)$ & $21(17 \%)$ & 217 & 8.30 & 0.004 \\
\hline \multicolumn{6}{|c|}{ Current complaints (some women had 2 or more complaints) } \\
\hline Itching & $48(52 \%)$ & $21(17 \%)$ & 219 & 30.28 & $<0.001$ \\
\hline Vulvar pain/soreness & $44(47 \%)$ & $15(12 \%)$ & 219 & 34.08 & $<0.001$ \\
\hline $\begin{array}{l}\text { Abnormal discharge } \\
\text { Vulvovaginal signs }\end{array}$ & $19(20 \%)$ & $22(17 \%)$ & 219 & 0.31 & NS \\
\hline Erythema & $50(54 \%)$ & $17(13 \%)$ & 219 & 40.87 & $<0.000$ \\
\hline Fissures & $28(30 \%)$ & $13(10 \%)$ & 219 & 13.77 & $<0.001$ \\
\hline Abnormal discharge & $39(42 \%)$ & $26(21 \%)$ & 218 & 12.03 & $<0.001$ \\
\hline
\end{tabular}

Table 3 Sexual and hygiene habits and associations to growth of candida

\begin{tabular}{|c|c|c|c|c|c|}
\hline & \multicolumn{2}{|l|}{ Candida } & \multirow[b]{2}{*}{ Number of answers } & \multirow[b]{2}{*}{$\chi^{2}$} & \multirow[b]{2}{*}{ p Value } \\
\hline & Positive & Negative & & & \\
\hline Frequent oro-genital sex & $46(50 \%)$ & $42(34 \%)$ & 214 & 5.81 & $<0.02$ \\
\hline Frequent intercourse* & $36(42 \%)$ & $35(33 \%)$ & 192 & 1.89 & NS \\
\hline Regular sex for $>4$ years & $20(22 \%)$ & $34(27 \%)$ & 214 & 0.75 & NS \\
\hline Current use of $O C$ & $75(82 \%)$ & $92(74 \%)$ & 216 & 2.33 & NS \\
\hline Ever use of $O C$ & 85 (93\%) & $110(88 \%)$ & 216 & 1.75 & NS \\
\hline OC for 3 years or longer & $36(39 \%)$ & $54(44 \%)$ & 216 & 0.42 & NS \\
\hline Sweets ( $>3$ times a week) & $22(24 \%)$ & $45(36 \%)$ & 216 & 3.44 & (0.06) \\
\hline Smoking (>10 cigarettes/day) & $10(11 \%)$ & $16(13 \%)$ & 208 & 0.23 & NS \\
\hline Pads between periods $†$ & $14(15 \%)$ & $21(17 \%)$ & 212 & 0.11 & NS \\
\hline Thongst & $58(64 \%)$ & $90(73 \%)$ & 215 & 1.91 & NS \\
\hline Shaving the pubic hairt & $57(63 \%)$ & $69(56 \%)$ & 214 & 1.27 & NS \\
\hline
\end{tabular}

Women with recurrent candidiasis are often advised to eliminate sweets and food rich in carbohydrates. ${ }^{20}$ According to our result there was a trend towards a relation between positive candida culture and frequent consumption of sweets.

Chronic candidiasis may be an initiating factor for $\mathrm{VVS}^{41}$ In our study, a history of recurrent candidiasis was significantly associated with frequent painful intercourse. Recurrent candidiasis in combination with OC use might strain the sensitive vestibular mucosa. It was recently observed that regular intercourse and/or use of OC before 16 years of age and for more than 2 years was significantly associated with pain at intercourse.22 ${ }^{23}$ A high dose of progesterone for contraceptive purposes induces a slight thinning of the vaginal epithelial layer and a decreased amount of hydrogen peroxide-producing lactobacilli. ${ }^{24}$ In the long run, this situation may affect the superficial nerve endings, which have been shown to overreact on mechanical stimuli in women with vulvar vestibulitis. ${ }^{25}$

In conclusion, the present work shows that according to culture, candida was present in $42 \%$ of sexually active women undergoing a genital examination at an adolescent health centre. Both positive candida culture and history of recurrent candidiasis were associated with vulvar pain at intercourse. Growth of candida was associated with oro-genital sex.

\section{ACKNOWLEDGEMENTS}

The authors acknowledge the midwives Eva-Marie Wenneberg, IngaLill Wiklund, and Barbro Eriksson at the adolescent centre for their assistance in collecting the material.

\section{CONTRIBUTORS}

ER initiated the study and has participated in the examination of the material as well as the completion of the manuscript; A-LB participated in the collection of material and the completion of the manuscript; CK participated in the organisation of the study as well as the examination and completion of the manuscript; BP provided facilities for the analyses of the material.

\section{Authors' affiliations}

E Rylander, C Krassny, Division of Obstetrics and Gynaecology, Karolinska Institutet Danderyd Hospital, Stockholm, Sweden A-L Berglund, Division of Health and Caring Sciences, Karlstad University, Karlstad, Sweden

B Petrini, Department of Clinical Microbiology, Karolinska Institutet, Karolinska Hospital, Stockholm, Sweden

\section{REFERENCES}

1 Goetsch MF. Vulvar vestibulitis: prevalence and historic features in a general gynecologic practice population. Am J Obstet Gynecol 1991;164:1609-17.

2 Danielsson I. Dyspareunia in women with special reference to vulvar vestibulitis. Medical Thesis. Umeå University, Sweden, 2001.

3 Friedrich EG. Vulvar vestibulitis syndrome. J Reprod Med 1987;32:110-14.

4 Baggish MS, Miklos JR. Vulvar pain syndrome: a review. Obstet Gynecol Surv 1995:50:618-22.

5 Foxman B. Epidemiology of vulvovaginal candidiasis: risk factors. Am J Public Health 1990:80:329-31.

6 Denning DW, and the Working Group of the British Society for Medical Mycology, et al. Management of genital candidiasis. BMJ 1995;310:1241-4.

7 Ferrer J. Vaginal candidosis: epidemiological and etiological factors. Int J Gynecol Obstet 2000;71:S21-7.

8 Adad SJ, de Lima RV, Sawan ZT, et al. Frequency of Trichomonas vaginalis, Candida sp and Gardnerella vaginalis in cervical-vaginal smears in four different decades. Sao Paulo Med J 2001;119:200-5.

9 Odds FC, Bernaerts R. CHROMagar Candida, a new differentiation medium for presumptive identification of clinically important Candida species. J Clin Microbiol 1994;32:1923-9.

10 Eckert LO, Hawes SE, Stevens CE, et al. Vulvovaginal candidiasis: clinical manifestations, risk factors, management algorithm. Obstet Gynecol 1998; $92: 757-65$.

11 Odds FC. Factors that predispose the host to candidosis. In: Odds FC, ed. Candida and candidosis. London: Baillière Tindall, 1988:94-114.

12 Jonsson $M$, Karlsson R, Rylander $E$, et al. The silent suffering woman-a population based study on the association between reported symptoms and 
past and present infections of the lower genital tract. Genitourin Med 1995;71:158-62.

13 Otero L, Palacio V, Carreno F, et al. Vulvovaginal candidiasis in female sex workers. Int J STD AIDS 1998:9:526-30.

14 Geiger AM, Foxman B. Risk factors for vulvovaginal candidiasis: a case-control study among university students. Epidemiology 1996;7:182-7.

15 Markos AR, Wade AAH, Walzman M. Oral sex and recurrent vulvo-vaginal candidiasis. Genitourin Med 1992;68:61-2.

16 Oates JK. Recurrent vaginitis and oral sex. Lancet 1979;1:785.

17 Reed BD, Gorenflo DW, Gillespie BW, et al. Sexual behaviour and other risk factors for Candida vulvovaginitis. J Womens Health Gend Based Med 2000;9:645.

18 Horowitz B, Edelstein SW, Lippman L. Sexual transmission of candida. Obstet Gynecol 1987;69:883-6.

19 Spinillo A, Capuzzo E, Nicola S, et al. The impact of oral contraception on vulvovaginal candidiasis. Contraception 1995;51:293-7.
20 Sobel JD, Faro S, Force RW, et al. Vulvovaginal candidiasis: epidemiologic, diagnostic, and therapeutic considerations. Am J Obstet Gynecol 1998;178:203-211.

21 Geiger AM, Foxman B, Sobel JD. Chronic vulvovaginal candidiasis: characteristics of women with Candida albicans, $C$ glabrata, and no candida. Genitourin Med 1995;71:304-7.

22 Berglund A-L, Nigard L, Rylander E. Vulvar pain, sexual behavior and genital infections in a young population. A pilot study. Acta Obstet Gynecol Scand 2002;81:738-42.

23 Bouchard C, Brisson J, Fortier M, et al. Use of oral contraceptive pills and vulvar vestibulitis: a case-control study. Am J Epidemiol 2002;156:254-61

24 Miller L, Patton DL, Meier A, et al. Depomedroxyprogesterone-induced hypoestrogenism and changes in vaginal flora and epithelium. Obstet Gynecol 2000;96:431-9.

25 Bohm-Starke N, Hilliges M, Brodda-Jansen G, et al. Psycho-physical evidence of nociceptor sensitization in vulvar vestibulitis syndrome. Pain 2001;94:177-83.

\section{CHESTER CHRONICLES}

\section{Loitering in Leeds}

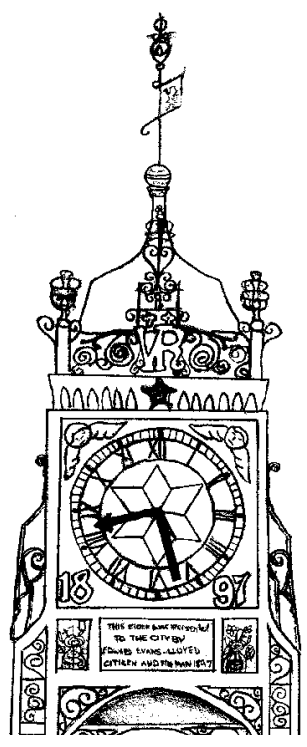

1 I s thee going in or staying out love?" "I'm not sure myself yet," says I—surprised to be asked to make a decision so early on in the day. "Well, no loitering here love, let thee make thy mind up." All I wanted to do was hover undecidedly at the back of the vast ornate lecture hall in Leeds Town Hall. I was only mildly interested in the topic about to be addressed and I needed thirty seconds or so to decide if the speaker had that certain ephemeral quality that makes the talk worth listening to. However, the calendar girls posted around the entrances were having none of it. Once the talk commenced the doors were bolted shut and that was it.

On the face of it, that might seem like a good idea for conference organisers but, in reality, imprisoning an audience isn't conducive to attentive listening. Look at the example of our European colleagues. At the last EADV in Prague, and indeed at the EADV Spring meeting, the audience could best be described as fluid. With parallel sessions happening all over the place, people would dip in and out of various symposia and workshops, maximising their time at the conference and also allowing some time for recovery and discourse with colleagues in the corridors and coffee areas. Easy access at the back and sensible seating layout meant that all seats were accessible without abseilling over the inevitable lone delegate sitting at the end of the row!

So what's the idea of a conference anyway? To educate and impart knowledge? Maybe-but for the likes of me working at the coal face in a district general hospital, the main purpose of attending a conference is to restore morale. I need my spirits uplifted and my enthusiasm rekindled. I get this by meeting colleagues and friends that I know are doing the same type of work as me, and we can all have a collective moan about how undervalued we are, and we feel all the better for this catharsis. The MSSVD Spring Conference does bring together a great number of like minded people, and most of the excitement and benefit of the conference comes not in the darkened lecture theatre, but in the corridors, pubs, and hotels of the conference venue. It is therefore critical that a good deal of time at a conference is set aside for networking, as opposed to formal structured lecturing. Some programmes are so full that a conference might just as well have been held in a motel off the M6, as opposed to a beautiful city like Leeds, for all you get to see of it.

So roll on the beautiful Roman city of Bath and the first BASHH/ASTDA conference in May 2004. Let's have late starts and early finishes, short sessions (parallel), and long breaks. Julius Caesar said "veni, vidi, vici," so let's hope there's plenty of "vidi" time for the legions who descend on the Spring MMIV.

C O'Mahony

Countess of Chester Hospital, Chester CH2 1UL, UK; colm.omahony@coch.nhs.uk 